\title{
Controlled tuning of atomistic structure in functional materials by acoustic standing waves and electric fields
}

\author{
C. Ludt ${ }^{1}$, E. Ovchinikova ${ }^{2}$, A. Kulikov ${ }^{3}$, D. Novikov, D.C. Meyer ${ }^{1}$, M. Zschornak ${ }^{1}$ \\ ${ }^{1}$ Institute of Experimental Physics, Technische Universität Bergakademie Freiberg, Leipziger Str. 23, 09599 Freiberg, Germany, \\ ${ }^{2}$ Physics Department, Moscow State University, 119991 Moscow, Russia, ${ }^{3}$ A.V. Shubnikov Institute of Crystallography, FSRC \\ “Crystallography and Photonics" RAS, Leninskiy Prospekt, 59, 119333 Moscow, Russia, ${ }^{4}$ Deutsches Elektronen-Synchrotron DESY, \\ Notkestraße 85, 22607 Hamburg, Germany
}

\section{Christian.Ludt@physik.tu-freiberg.de}

Active research in the field of condensed matter and nanotechnology not only led to significant progress in understanding the mechanisms of formation of electrical polarization and magnetoelectric phenomena, but also showed the possibilities of creating new classes of devices based on a combination of magnetoelectric and piezoelectric properties. Meanwhile, macroscopic properties, such as multiferroism and piezoelectricity, are associated with local structural changes that occur under the influence of external perturbations. In a first step chosen crystal structures are analyzed by means of density functional theory (DFT) to validate the connection of external stress and internal change of lattice symmetry as well as atomic displacements. Among them are $\mathrm{TeO}_{2}, \mathrm{Li}_{2} \mathrm{~B}_{4} \mathrm{O}_{7}, \mathrm{ZnO}$ and $\mathrm{SrTiO}$. Also in focus is the influence of oxygen vacancies on our structures. The research is currently accompanied by experiments in which standing acoustic waves are encoupled in crystal samples to change the structure parameters and particularly the structures' symmetry locally. Because the displacements are expected to be on the picometer scale, X-ray diffraction on forbidden reflections is applied to observe the induced effects. The obtained switching results can significantly widen the range of functional materials and can be directly used in modern technological applications.

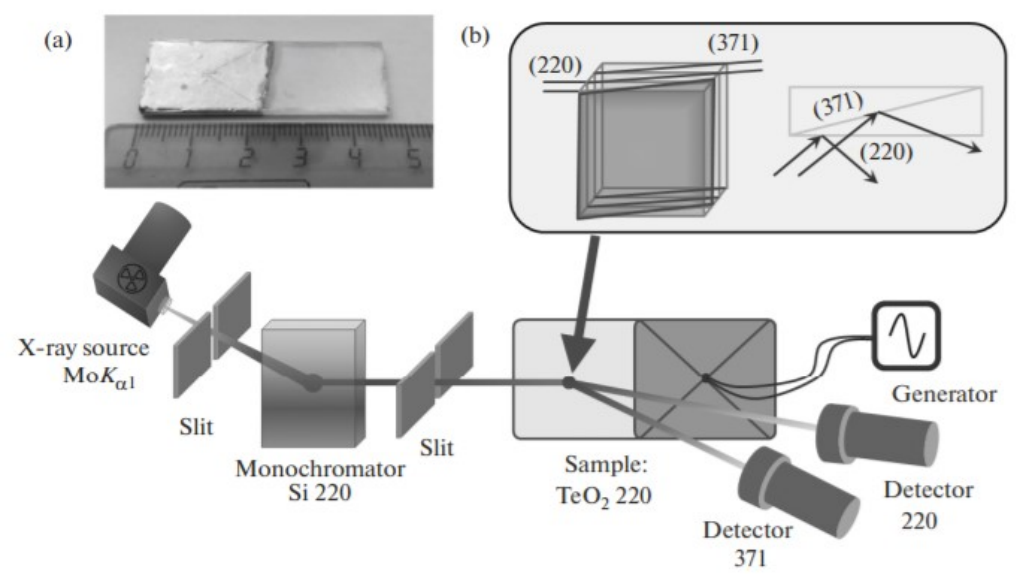

Figure 1. (a) The sample is an X-ray acoustic resonator based on a paratellurite crystal. (b) Double-crystal parallel circuit for conducting multiple-wave diffraction studies, assembled on the basis of a TRS-1 diffractometer [1].

[1] Eliovich et al. „Multiple-Wave Interaction of X-Rays in a Paratellurite Crystal under Excitation of Ultrasonic Vibrations“. J. Synch. Investig. 13, 570-577 (2019)

Keywords: functional materials, tunable properites, DFT

C.L. and M.Z. acknowledge funding by the DFG within the projects DFG 409743569, ZS 120/1-1 and DFG 405595647. E.O. and A.K. acknowledge further funding by the Russian Foundation for Basic Research (project No. 19-52-12029a). 